\title{
28 Research Square \\ The Association of Wolbachia on the Gene Expression in Drosophila Adult Testis
}

\author{
Wei-Hao Dou \\ College of Life Science, Nankai University \\ Jin-Hua Xiao ( $\nabla$ xiaojh@nankai.edu.cn ) \\ https://orcid.org/0000-0001-6105-8976 \\ Da-Wei Huang \\ College of Life Science, Nankai University
}

\section{Research article}

Keywords: Drosophila melanogaster, Wolbachia, Lipid metabolism, Carbohydrate metabolism, Intracellular symbionts

Posted Date: July 9th, 2020

DOl: https://doi.org/10.21203/rs.3.rs-39431/v1

License: (c) (i) This work is licensed under a Creative Commons Attribution 4.0 International License. Read Full License 


\section{Abstract}

Background: Wolbachia is a type of intracellular symbiotic bacteria widely distributed in arthropods including most insects and nematodes. These maternally inherited bacteria can regulate the host's reproductive system in various ways for their own vertical transmission. Since the identification of Wolbachia in many insects, the relationship between Wolbachia and host has attracted great interest. Wolbachia must rely on the host cells to survive, and they can also improve the fitness of the host through a variety of ways. However, the molecular basis of interaction between Wolbachia and their host has not been well resolved so far.

Results: We performed transcriptome sequencing on testis tissues of adults of Wolbachia-infected and Wolbachia-free Drosophila melanogaster. Comparison of gene expression profiles revealed 471 significantly differentially expressed genes that involved in cell metabolism, cell membrane component correlation and hydrolysis process.

Conclusions: Our results show that lipid and carbohydrate metabolism are more active in Wolbachiainfected testis than in Wolbachia free testis. This work strengthens our general understanding of the Wolbachia-host intracellular relationship and may provide a new perspective for Wolbachia-mediated virus-blocking.

\section{Background}

Wolbachia is a class of intracellular bacteria that are widely spread in arthropods and nematodes, and this maternally transmitted bacteria can regulate the host's reproductive system in a variety of ways to increase its chances of transmission, such as cytoplasmic incompatibility $(\mathrm{Cl})$, parthenogenesis inducing $(\mathrm{PI})$, male killing (MK), femalization and enhancement of female fertility [1-4],and these manipulations are considered as a kind of "selfish strategies" to increase their infection rate in the host population and reduce host fitness $[1,5]$. But in other ways Wolbachia can significantly improve host fitness. For example, Wolbachia is necessary for nematode growth and development, as the adult worms were retarded, ovaries degenerated, and embryos could not form when Wolbachia was remove from nematodes by antibiotics [6]. In insect hosts, Wolbachia can increase the host's fertility [7-11], and enhance the insect host's resistance to many pathogens (such as RNA virus) [12-14]. Wolbachia can also provide vitamins to the host and give the host a clear growth advantage in the face of adversity [1, 15]. In addition, the host longevity, olfactory response, immunity and stem cell proliferation were also affected by Wolbachia [16-18]. It is expected that Wolbachia have evolved mutualistic relationship with many of their hosts [19].

Wolbachia infect a remarkable range of insect hosts suggests their interaction with various host metabolic pathways for their successful intracellular maintenance within a host [20]. This interactive relationship is complex and has received extensive attention. Recent transcriptomic, genomic, and proteomic data have revealed that Wolbachia affects many physiological processes in the host. Previous 
studies that combined genome-wide RNAi screening and new high-throughput fluorescence in situ hybridization technology in Drosophila cell lines have found that a large number of host genes may be involved in the alteration of Wolbachia level. These genes are involved in lipid metabolism, transport, protein degradation, translation, and cell cycle [21, 22], indicating that many host's metabolic pathways have an obvious impact on the survival of Wolbachia. A genome-wide analysis of various insect-related Wolbachia strains has indicated that Wolbachia has a complete and conserved glycolysis pathway and riboflavin (vitamin B2) synthesis pathway [23]. Riboflavin synthesized by Wolbachia has a significant contribution to the growth and development of bed bugs [15]. However, Wolbachia is highly dependent on the host carbon source (such as phosphoglyceride), amino acids (such as alanine) and phospholipids [23]; Wolbachia lacks lipid metabolism genes, especially lipid A synthesis genes [24], so Wolbachia must obtaining these lipids from the host to support cell growth. In addition, the proliferation of Wolbachia in the host is cholesterol-dependent, and Wolbachia lacks cholesterol synthesis genes, so their survival is likely related to the transport of host cholesterol. A recent study also showed that Wolbachia can be localized in the endoplasmic reticulum, which is the main site of lipid metabolism, affecting the distribution of the endoplasmic reticulum and obtaining vacuole membranes from it [25]. Therefore, the above studies have indicated that Wolbachia has a close nutritional symbiosis relationship with the host, so lipids and carbohydrate metabolism is likely to play an extremely important role in the symbiotic relationship between Wolbachia and the host. However, the interaction between Wolbachia and the host in lipid and carbohydrate metabolism has not been studied in details.

Wolbachia is mainly localized in the reproductive tissues of host [26], which is a good material for studying the interaction between Wolbachia and host. Previous studies have showed that Wolbachia affects the early spermatogenesis process [27]. The gene expression pattern between the Drosophila melanogasterlarval testis in the Wolbachia-infected and the Wolbachia-free samples observed differential expression of genes related to metabolism, immunity, reproduction and other functions, some of which that may be related to spermatogenesis, including Ance, lola and Mst84Db, were down-regulated in Wolbachia-infected sample, inducing abnormal spermatogenesis that causes Wolbachia to "modify" sperm, which may be the cause of $\mathrm{Cl}$. However, in Drosophila, mature sperms are continuously produced in the testis after eclosion but not in the larval stage [28]. Therefore, the purpose of this study is to explore the putative effect of Wolbachia in the process of sperm maturation, based on the gene expression in the testis tissue of Drosophila adults.

We selected Wolbachia-infected and Wolbachia-free Drosophila adult testis tissues for RNA-seq, and analyzed the differentially expressed genes. A total of 472 genes are different expressed at least a 2 fold change (q-value $<0.1 \%$ ) between Wolbachia-infected and Wolbachia-free testis, involving a variety of physiological processes such as innate immune immunity, carbohydrate metabolism, and lipid metabolism. Most of the differentially expressed genes involved in innate immunity including Toll and IMD pathway were up-regulated in the presence of Wolbachia. More interestingly, lipid and carbohydrate metabolism related gene were significant up-regulated in the presence of Wolbachia, while epidermal wax ester synthesis related genes were down-regulated by Wolbachia. Our research found for the first time that the presence of Wolbachia is associated with more active lipid metabolism and carbohydrate 
metabolism in the host. These results can provide important evidence for elucidating the mechanism of Wolbachia-host intracellular relationship.

\section{Result}

\section{Transcriptome sequencing data of Drosophila adult testis}

We performed transcriptome sequencing on testis tissues of adults (one day after eclosion) of Wolbachia-infected (WInM) and Wolbachia-free (WUnM) Drosophila melanogaster. Comparison of gene expression profiles revealed 471 significantly differentially expressed genes (at least $2 \mathrm{fold}$, q-value < $0.1 \%)$, with 402 genes up-regulated and 69 genes down-regulated in the presence of Wolbachia infection.

The significantly differentially expressed genes between Wolbachia-infected (WInM) and Wolbachia-free (WUnM) samples are involved in many metabolic pathways

We performed Gene Ontology classification analysis on the 472 significantly differentially expressed genes and found that they were involved in cell metabolism, cell membrane component correlation and hydrolysis process (Fig. 1a, b). Results of KEGG enrichment analysis showed that up-regulated genes in WInM were mostly involved in transport, signal transduction, immune response, glucose metabolism, lipid metabolism and digestion processes, while down-regulated genes in WInM was involved in the insect epidermal wax ester synthesis pathway (Fig. 2).

We detected altogether six significantly up-regulated genes in WInM were involved in the innate immune pathway of the Drosophila (Table 1 \& Fig. 3a), all of which including DptB, CG9673, spheroide, Tak/1, $D r s / 3$, and the peptidoglycan recognition protein gene PGRP-SC2 were functional in Toll and IMD pathways. 
Table 1

Genes related to immune response that are differentially expressed ( $\geq 2$ fold changes, q-value $<0.1 \%$ ) in testes of Wolbachia-infected flies compared to Wolbachia-free flies.

\begin{tabular}{|c|c|c|c|}
\hline $\begin{array}{l}\text { Gene } \\
\text { Symbol }\end{array}$ & $\begin{array}{l}\log 2 \\
(W \ln M / W U n M)\end{array}$ & KEGG Pathway & Biological Process \\
\hline DptB & 1.41 & $\begin{array}{l}\text { Toll and Imd signaling } \\
\text { pathway }\end{array}$ & $\begin{array}{l}\text { defense response to Gram-negative } \\
\text { bacterium }\end{array}$ \\
\hline CG9673 & 8.09 & $\begin{array}{l}\text { Toll and Imd signaling } \\
\text { pathway }\end{array}$ & proteolysis \\
\hline \multirow[t]{2}{*}{ spheroide } & 8.48 & $\begin{array}{l}\text { Toll and Imd signaling } \\
\text { pathway }\end{array}$ & $\begin{array}{l}\text { positive regulation of Toll signaling } \\
\text { pathway, }\end{array}$ \\
\hline & & & $\begin{array}{l}\text { defense response to Gram-positive } \\
\text { bacterium }\end{array}$ \\
\hline Takl1 & 2.02 & $\begin{array}{l}\text { Toll and Imd signaling } \\
\text { pathway }\end{array}$ & defense response to fungus \\
\hline Drsl3 & 1.88 & $\begin{array}{l}\text { Toll and Imd signaling } \\
\text { pathway }\end{array}$ & $\begin{array}{l}\text { phosphorylation,activation of MAPKK } \\
\text { activity }\end{array}$ \\
\hline $\begin{array}{l}\text { PGRP- } \\
\text { SC2 }\end{array}$ & 1.54 & & $\begin{array}{l}\text { negative regulation of innate immune } \\
\text { response }\end{array}$ \\
\hline
\end{tabular}

Genes related to carbohydrate metabolism, especially the genes related to the hydrolysis of sucrose and starch, including Mal-A1, Mal-A3 and Mal-A4, were significantly up-regulated in Wolbachia-infected Drosopshila testis. Among them, Mal-A1 was up-regulated in Wolbachia infected testis by nearly 700 folds (Fig. 3b). In addition, the expression of UDP-glycosyltransferase genes such as CG5724, CG5999, Ugt86Dh were also significantly up-regulated in Wolbachia-infected testis (Fig. 3d), and these genes are necessary for Wolbachia in the synthesis of LipidA and further synthesis of lipopolysaccharide (LPS) [29].

We also found that the several genes involved in lipid metabolism were significantly up-regulated in Wolbachia-infected Drosophila adults testis, among which the genes of mag and CG10116 were involved in lipid degradation, genes of CG5804 and CG8628 encoded acetyl-CoA binding proteins, gene of CG8834 was related in fatty acid synthesis, gene of $A c o x 57 D-d$ was functional in fatty acid degradation, and genes of $N p c 2 f$ and $N p c 2 d$ were involved in intracellular cholesterol transportation (Table 2 \& Fig. 3c). In contrast, the genes of CG10097, CG1441, CG13091, and CG17560, which were involved in the synthesis of other lipid derivatives such as fatty alcohols from fatty acid, were significantly down-regulated in Wolbachia-infected testis, which were annotated to have fatty acyl-CoA reductase (alcohol-forming) activity, or be involved in the biosynthesis of insect cutin and wax (Table 2). 
Table 2

Classification of genes related to Lipid metabolism that are differentially expressed ( $\geq 2$ fold changes, qvalue $<0.1 \%$ ) in testes of Wolbachia-infected flies compared to uninfected flies .

\begin{tabular}{|c|c|c|c|c|}
\hline & $\begin{array}{l}\text { Gene } \\
\text { Symbol }\end{array}$ & $\begin{array}{l}\log 2 \\
\text { (WInM/WUnM) }\end{array}$ & GO analysis & KEGG pathway \\
\hline \multirow{17}{*}{$\begin{array}{l}\text { Lipid } \\
\text { metabolism }\end{array}$} & CG5804 & 9.67 & fatty-acyl-CoA binding & \\
\hline & CG8628 & 4.29 & fatty-acyl-CoA binding & \\
\hline & CG10116 & 6.63 & lipid catabolic process & \\
\hline & mag & 5.84 & lipid metabolic process & \\
\hline & $\mathrm{Npc} 2 \mathrm{f}$ & 1.33 & $\begin{array}{l}\text { intracellular cholesterol } \\
\text { transport }\end{array}$ & \\
\hline & $\mathrm{Npc} 2 \mathrm{~d}$ & 4.33 & $\begin{array}{l}\text { intracellular cholesterol } \\
\text { transport }\end{array}$ & \\
\hline & $\begin{array}{l}\text { Acox57D- } \\
d\end{array}$ & 1.66 & fatty acid beta-oxidation & Fatty acid degradation \\
\hline & CG8834 & 3.08 & $\begin{array}{l}\text { fatty acid biosynthetic } \\
\text { process }\end{array}$ & \\
\hline & CG9084 & 3.53 & $\begin{array}{l}\text { phospholipid scramblase } \\
\text { activity }\end{array}$ & \\
\hline & CG1441 & -1.08 & $\begin{array}{l}\text { alcohol-forming fatty acyl- } \\
\text { CoA }\end{array}$ & $\begin{array}{l}\text { Cutin and wax } \\
\text { biosynthesis }\end{array}$ \\
\hline & & & reductase activity & \\
\hline & CG13091 & -1.16 & $\begin{array}{l}\text { alcohol-forming fatty acyl- } \\
\text { CoA }\end{array}$ & $\begin{array}{l}\text { Cutin and wax } \\
\text { biosynthesis }\end{array}$ \\
\hline & & & reductase activity & \\
\hline & CG10097 & -1.30 & $\begin{array}{l}\text { alcohol-forming fatty acyl- } \\
\text { CoA }\end{array}$ & $\begin{array}{l}\text { Cutin and wax } \\
\text { biosynthesis }\end{array}$ \\
\hline & & & reductase activity & \\
\hline & CG17560 & -1.24 & $\begin{array}{l}\text { alcohol-forming fatty acyl- } \\
\text { CoA }\end{array}$ & $\begin{array}{l}\text { Cutin and wax } \\
\text { biosynthesis }\end{array}$ \\
\hline & & & reductase activity & \\
\hline
\end{tabular}

\section{Discussion}

Wolbachia and its host have a long history of co-evolution, and the interaction between both parters is very complex, which has not been clearly clarified so far [30]. Wolbachia is mainly located in the insect host reproductive system [26], including female ovary and male testis which makes them good materials for studying Wolbachia-host interaction. Previous results based on the transcriptomic data of the testis 
tissue of the third instar larvae of Drosophila have shown that Wolbachia infection can affect the expression of genes related to spermatogenesis and thus may induce $\mathrm{Cl}$ [27]. In this study, we focused on the gene expression in the adult testes of the Wolbachia-infected and Wolbachia-free Drosophila melanogaster to investigate the possible effects of Wolbachia on the host reproductive system during the process of sperm maturity. The results showed that the expression of genes involved in innate immune system and multiple metabolic pathways, especially lipid and carbohydrate metabolism, were significantly different between Wolbachia-infected and Wolbachia-free Drosophila adult testis. We speculate that Wolbachia may competes with its host for carbohydrate and lipid metabolism resources, on the other hand, Wolbachia also provides vitamin for the host (Fig. 4).

\section{Wolbachia is associated with the high expression of innate immune genes in native host}

When insects are infected by pathogenic bacteria, the host innate immune responses such as Toll and IMD signaling pathways are activated and then produce a variety of antibacterial peptides (AMPs) [31]. Wolbachia can survive in host cell, and some data have shown that it does not induce immune responses in its native host $[12,32-34]$. However, our results showed that Wolbachia was related in enhanced immune responses in its native host testis, including multiple genes in the Toll and IMD pathways, such as DptB, CG9673, spheroide, Tak/1 and Drs/3. For example, the protein encoded by the DptB gene is an antimicrobial peptide induced by the IMD signal pathway, which is specifically produced in insect fat bodies and can resist gram-negative bacteria infection [35], indicating that Wolbachia, as a gram-negative bacteria, can still induce the immune response in its native host. In addition, spheroide and Tak/1 are usually involved in activating Toll signaling pathways in resisting gram-positive bacteria and fungal infections [36]. It can be seen that in the naturally infected host, Wolbachia may still lead to an increase in its immune response. The enhanced host's immune system may be a "double-edged sword" for Wolbachia, which is harmful to itself and limits infection with other pathogenic bacteria, prevents the pathogenic bacteria for snatching intracellular resources. Of course, Wolbachia may also escape the host 's immune system in various ways. The expression level of peptidoglycan recognition protein PGRP-SC2 is significantly higher in Wolbachia infected testis, PGRP-SC2 can negatively regulate the IMD signaling pathway by hydrolyzing peptidoglycan, preventing the activation of the constitutive IMD pathway, thereby maintaining the balance between immune tolerance and immune response for Wolbachia infection [37]. Our subsequent transcriptome data (unpublished) shows that the expression levels of Toll and IMD pathway related genes are not different between infected and uninfected Drosophila female ovaries, so it can be speculated that the relationship between Wolbachia and male or female hosts is different. Wolbachia may escape the host's immune system in other ways in females, for example, some studies have pointed out that Wolbachia itself can encode Peptidoglycan Amidase to avoid the host's immune system [38].

\section{Wolbachia is associated with the more active carbohydrate metabolism process in the host}

In this study, we noticed that the carbohydrate metabolism was more active in the Wolbachia-infected sample, which might be associated with the infection of Wolbachia. First, the expression levels of Mal-A1, 
$M a l-A 3$, and $M a l-A 4$ genes related to starch and sucrose hydrolase activity were significantly up-regulated in Wolbachia infected Drosophila testis. These genes can accelerate the formation of D-glucose, which is the initial substrate of glycolysis. It has been reported that insect associated Wolbachia exhibits a complete glycolysis metabolic pathway [23], so it is possible that Wolbachia compete with the host to consume the glycolysis substrate-glucose, resulting in a more active sugar metabolism in the host.

Second, the expression levels of UDP-glycosyltransferase genes including CG5724, CG5999, and Ugt86Dh were significantly up-regulated in Wolbachia-infected testes. Actually, there is no gene involved in UDPglycosylation in the Wolbachia genome [23], even though Wolbachia need this function. Wolbachia must synthesize its own cell wall and Lipopolysaccharides (LPS) is an essential component of the cell wall [11]. The glycosylation reaction is a very important step in the biosynthesis of LPS, in which the host glycosyltransferase plays vital function for Wolbchia to survive [39]. Our results that the glycosyltransferase genes were up-regulated in the Wolbachia-infected host indicated that Wolbachia might heavily rely on the host in the process of LPS synthesis.

\section{Wolbachia is associated with the more active lipid metabolism in the host}

We detected significantly differentially expressed genes in lipid metabolism between Wolbachia-infected and Wolbachia-uninfected Drosophila testes. Two genes including CG5804 and CG8628 were significantly up-regulated in Wolbachia-infected Drosophila testis, both of which belonged to the acetylCoA binding protein (ACBP) family which were involved in regulating the expression of genes related to lipid metabolism. Interestingly, we detected that lipolysis-related genes such as mag and CG10116 were also up-regulated in Wolbachia-infected Drosophila testis, and this genes can regulate the storage of triacylglycerol (TAG) and maintain the balance of fat metabolism [40, 41]. Finally, the expression levels of genes $\mathrm{Npc2f}$ and $\mathrm{Npc2d}$ involved in intracellular cholesterol transport were also significantly up-regulated in Wolbachia-infected testis. All of these results indicated that the existence of Wolbachia was related to the high expression of genes related to lipid metabolism and transport in the host, suggesting that it may induce a more active fat metabolism in the host.

On the contrary, a gene (CG10097) in lipid derivatives synthesis was significantly down-regulated in Wolbachia-infected Drosophila testis tissue. Both GO molecular functional analysis and KEGG analysis showed that this gene encoded fatty acyl-CoA reductase (alcohol-forming), which was the key enzyme in the biosynthesis of insect epidermal wax esters or insect cutin and wax. This indicated that during the period of proliferation of Wolbachia in the host, the host might concentrate fatty acid resources for necessary lipid metabolism for survival, which thus restricted its conversion process to other lipid derivatives.

\section{Conclusions}

Wolbachia and the insect host has had a long co-evolutionary history that formed a mutually beneficial symbiosis. Wolbachia provides the necessary nutrients for the host, and its survival is strictly dependent on the host. Our RNA-seq data based on Drosophila adult testis indicated that Wolbachia may affect 
various physiological pathways of the host, such as immunity, glucose metabolism and lipid metabolism. These data provide important molecular evidence for the Wolbachia-host intracellular relationship. Subsequent analysis of transcriptome data in Drosophila ovaries may help further understand the differences in Wolbachia-host molecular interactions between male and female hosts.

\section{Methods}

\section{Fruit fly rearing}

Drosophila melanogaster in this laboratory was kindly donated by Prof. Hu Haoyuan in Anhui Normal University. The standard medium of Drosophila corn flour was used for feeding. $60 \mathrm{~g}$ corn flour, $30 \mathrm{~g}$ brown sugar, $5 \mathrm{~g}$ sucrose, $1 \mathrm{~g}$ sodium benzoate, $6 \mathrm{~g}$ agar powder, $800 \mathrm{ml}$ water was added and boiled for $5 \mathrm{~min}$. After cooling, $5 \mathrm{~g}$ yeast powder was added to make fruit fly medium. The fruit flies were reared in an artificial climate box (Ningbo Jiangnan Instrument Factory), light: dark $=14 \mathrm{~L}: 10 \mathrm{D}$, relative humidity $40 \%$, light 7000Lux [42].

\section{Wolbachia -free fruit fly strains}

We used the MLST method to detect the Wolbachia strain in fruit flies [43] and found that the Drosophila melanogaster naturally infected the Wolbachia wMel strain, and the Wolbachia-infected Drosophila melanogaster strain was named WIn. We prepared tetracycline stock solution $10 \mathrm{mg} / \mathrm{mL}$, adding $2 \mathrm{~mL}$ tetracycline stock solution per $100 \mathrm{~g}$ corn flour medium [44]. After continuous treatment for three generations, by PCR detection of Wolbachia wsp, ftsz, and $16 \mathrm{~s}$ genes, we obtained Wolbachia-free fruit fly strain WUn and then transferred them to standard medium and continuously cultivated for more than 5 generations to remove the effects of antibiotics.

\section{RNA-seq}

The WIn and WUn male fruit flies that had been reared in corn flour medium for one day after eclosion then dissected in RNase-free water, and the complete testis was placed in RNAhold and stored at $-80^{\circ} \mathrm{C}$. Total RNA was extracted by using TansZo/Up Plus RNA Kit, about 10 Drosophila testes were used for each sample. Using PE150bp pair-end transcriptome sequencing in BGISEQ-500 platform (BGI, Shenzhen, China), the amount of sequencing data requires $6 \mathrm{G}$ for each sample. The sequnces were submitted to NCBI with the accession number of PRJNA639180.

\section{Quantitative real-time PCR (qPCR)}

To further investigate the differentially expressed genes identified by RNA-seq, 17 genes were selected for qRT-PCR analysis. Specific primers for the 17 genes and RP49 (Ribosome protein 49 reference gene) were designed by NCBI primer-BLAST (Additional file 1).

RNA extraction from WIn and WUn testis was carried out using TransZol Up Plus RNA Kit (TransGen, Beijing, China) according to the instructions, RNA reverse transcription was performed using Transscript 
One-Step gDNA Renover and cDNA Synthsis SuperMix (TransGen, Beijing, China), $1 \mu \mathrm{g}$ RNA was used for reverse transcription, gDNA remover $1 \mu \mathrm{L}$, Oligo(dT) $1 \mu \mathrm{L}$, 2xTS Reaction Mix 10 $\mathrm{L}$, TransScript RT/RI Enzyme Mix $1 \mu \mathrm{L}$, added Nuclease-free water for total volume $20 \mu \mathrm{L}$. Reaction conditions : $42^{\circ} \mathrm{C}$ for $30 \mathrm{~min}$, then $85^{\circ} \mathrm{C}$ for $5 \mathrm{sec}$. QPCR verifies differential gene expression. The primers were shown in Table S1. We used the $\Delta \Delta C$ t method, by using the PerfectStart Green qPCR SuperMix Kit (TransGen, Beijing, China), the reaction system is cDNA $1 \mu \mathrm{L}$, Forward/Reverse Primer each $0.4 \mu \mathrm{L}(10 \mu \mathrm{M} / \mathrm{L}), 2 x$ PerfectStart Green qPCR SuperMix $10 \mu \mathrm{L}$, and Nuclease-free water $8.2 \mu \mathrm{L}$. Reaction conditions: Pre-reaction at $94^{\circ} \mathrm{C}$ for $30 \mathrm{~s}$, then 40 cycles of $94^{\circ} \mathrm{C}$ for $5 \mathrm{~s}, 60^{\circ} \mathrm{C}$ for $30 \mathrm{~s}$, and then the dissolution step was performed.

\section{Data analysis}

For data analysis, multiple $t$ test in Graphpad prism8 was used to analyze the significance of differences between two groups. $p$-value of less than 0.05 was considered significant.

\section{Abbreviations}

WInM

Wolbachia-infected male; WUnM:Wolbachia-uninfected male; WIn:Wolbachia-infected Drosophila melanogaster strain; WUn: Wolbachia-uninfected Drosophila melanogaster strain.

LPS:Lipopolysaccharides; ACBP:Acetyl-CoA binding protein family; TAG:Triacylglycerol;

DHAP:Dihydroxyacetone phosphate.

\section{Declarations}

\section{Acknowlegements}

We would like to thank Haoyuan Hu for donating fruit fly, we also thank Dan Zhao for many insightful discussions on the obtained results. Sequencing was performed at the BGISEQ-500 platform in Shenzhen, China.

\section{Authors' contributions}

JHX and DWH conceived the study. WHD analysed the data, performed QPCR verification and wrote the paper. All authors have read and approved the manuscript.

\section{Funding}

This study was supported by the National Natural Science Foundation of China (No.s of 31830084, 31970440 \& 31672336), and also supported by the construction funds for the "Double First-Class" initiative for Nankai University (Nos. 96172158, 96173250 \& 91822294). The funders had no role in study design, data analysis and manuscript drafting.

\section{Availability of data and materials}


The datasets generated and analyzed during the current study are available in the SRA database at $\mathrm{NCBI}$, with the accession number of PRJNA639180.

\section{Ethics approval and consent to participate}

Not applicable.

\section{Consent for publication}

Not applicable.

\section{Competing interests}

The authors declare that they have no competing interest.

\section{References}

1. Werren JH, Baldo L, Clark ME. Wolbachia: master manipulators of invertebrate biology. Nat Rev Microbiol. 2008;6(10):741-51.

2. Serbus LR, Casper-Lindley C, Landmann F, Sullivan W. The Genetics and Cell Biology of WolbachiaHost Interactions. Annu Rev Genet. 2008;42(1):683-707.

3. Vandekerckhove TTM, Watteyne S, Bonne W, Vanacker D, Mertens J. Evolutionary trends in feminization and intersexuality in woodlice (Crustacea, Isopoda) infected with Wolbachia pipientis (a-Proteobacteria). Belgian Journal of Zoology. 2003;133(1).

4. Weeks AR, Breeuwer JA. Wolbachia-induced parthenogenesis in a genus of phytophagous mites. Proc Biol Sci. 2001;268(1482):2245-51.

5. Stouthamer R, Breeuwer JA, Hurst GD. Wolbachia pipientis: microbial manipulator of arthropod reproduction. Annu Rev Microbiol. 1999;53(53):71-102.

6. Foster JM, Hoerauf A, Slatko BE, Taylor MJ, Kennedy MW, Harnett W. The Wolbachia bacterial endosymbionts of filarial nematodes. Parasitic Nematodes Molecular Biology Biochemistry Immunology. 2013;60:308-36.

7. Guo Y, Hoffmann AA, Xu XQ, Zhang X, Huang HJ, Ju JF, Gong JT, Hong XY. Wolbachia-induced apoptosis associated with increased fecundity in Laodelphax striatellus (Hemiptera: Delphacidae). Insect Mol Biol. 2018;27(6):796-807.

8. Guruprasad NM, Mouton L, Puttaraju HP. Effect of Wolbachia infection and temperature variations on the fecundity of the Uzifly Exorista sorbillans (Diptera: Tachinidae). Symbiosis.

9. 2011:;54(3):151-158.

10. Harcombe W, Hoffmann AA. Wolbachia effects in Drosophila melanogaster: in search of fitness benefits. J Invertebr Pathol. 2004;87(1):0-50. 
11. Dong C, Zhang YJ, Bi YP, Fu HB. Wolbachia endosymbionts and their effects on the fitness of the arthropod hosts. Acta Microbiologica Sinica. 2005;45(5):817-20.

12. Ashby B, Boots M. Coevolution of parasite virulence and host mating strategies. PNAS. 2015;112(43):13290-5.

13. Liu QQ, Zhang TS, Li CX, Gu JW, Hou JB, Dong H. Decision-making in a bisexual line and a thelytokous Wolbachia-infected line of Trichogramma dendrolimi Matsumura (Hymenoptera: Trichogrammatidae) regarding behavior toward their hosts. Pest Manag Sci. 2018;74(7):1720-7.

14. Newton IL, Clark ME, Kent BN, Bordenstein SR, Qu J, Richards S, Kelkar YD, Werren JH. Comparative Genomics of Two Closely Related Wolbachia with Different Reproductive Effects on Hosts. Genome Biol Evol. 2016;8(5):1526-42.

15. Niu X, Sun Y, Chen Z, Li R, Padmanabhan C, Ruan J, Kreuze JF, Ling K, Fei Z, Gao S. Using Small RNAseq Data to Detect siRNA Duplexes Induced by Plant Viruses. Genes (Basel). 2017;8(6):163.

16. Moriyama M, Nikoh N, Hosokawa T, Fukatsu T. Riboflavin Provisioning Underlies Wolbachia's Fitness Contribution to Its Insect Host. Mbio. 2015;6(6):e01732-15.

17. Fast EM, Toomey ME, Panaram K, Desjardins D, Kolaczyk ED, Frydman HM. Wolbachia Enhance Drosophila Stem Cell Proliferation and Target the Germline Stem Cell Niche. Science. 2011;334(6058):990-2.

18. Maistrenko OM, Serga SV, Vaiserman AM, Kozeretska IA. Longevity-modulating effects of symbiosis: insights fromDrosophila-Wolbachiainteraction. Biogerontology, 17(5-6):785-803.

19. Peng Y, Wang YF. Infection of Wolbachia may improve the olfactory response of Drosophila. Chinese Science Bulletin. 2009;(08):89-95.

20. Zug R, Hammerstein P. Bad guys turned nice? A critical assessment of Wolbachia mutualisms in arthropod hosts. Biol Rev Camb Philos Soc. 2015;90(1):89-111.

21. Jose E. Pietri HDaWS. The rich somatic life of Wolbachia. Microbiologyopen. 2016;5(6):923-36.

22. Grobler $Y$, Yun CY, Kahler DJ, Bergman CM, Lee H, Oliver B, Lehmann R. Whole genome screen reveals a novel relationship between Wolbachia levels and Drosophila host translation. PLoS Pathog. 2018;14(11):e1007445.

23. White PM, Serbus LR, Debec A, Codina A, Sullivan W. Reliance of Wolbachia on High Rates of Host Proteolysis Revealed by a Genome-Wide RNAi Screen of Drosophila Cells. Genetics. 2017;205(4):1473-88.

24. Jimenez NE, Gerdtzen ZP, Olivera-Nappa A, Salgado JC, Conca C. A systems biology approach for studying Wolbachia metabolism reveals points of interaction with its host in the context of arboviral infection. PLoS Negl Trop Dis. 2019;13(8):e0007678.

25. Foster J, Ganatra M, Kamal I, Ware J, Makarova K, Ivanova N, Bhattacharyya A, Kapatral V, Kumar S, Posfai J. The Wolbachia Genome of Brugia malayi: Endosymbiont Evolution within a Human Pathogenic Nematode. Plos Biology. 2005;3(4):e121. 
26. Fattouh N, Cazevieille C, Landmann F. Wolbachia endosymbionts subvert the endoplasmic reticulum to acquire host membranes without triggering ER stress. PLoS Negl Trop Dis. 2019;13(3):e0007218.

27. Clark ME, Veneti Z, Bourtzis K, Karr TL. Wolbachia distribution and cytoplasmic incompatibility during sperm development: the cyst as the basic cellular unit of $\mathrm{Cl}$ expression. Mech Dev. 2003;120(2):185-98.

28. Zheng Y, Wang JL, Liu C, Wang CP, Wang YF. Differentially expressed profiles in the larval testes of Wolbachia infected and uninfected Drosophila. Bmc Genomics. 2011;12(1):595.

29. Spradling A, Fuller MT, Braun RE, Yoshida S. Germline Stem Cells. Cold Spring Harb Perspect Biol. 2011;3(11):a002642-2.

30. Bishop RE. Fundamentals of endotoxin structure and function. Contrib Microbiol. 2005;12:1-27.

31. Landmann F. The Wolbachia Endosymbionts. Microbiology spectrum. 2019;7(2).

32. Lemaitre B, Hoffmann J. The host defense of Drosophila melanogaster. Annu Rev Immunol. 2007;25:697-743.

33. Wong ZS, Hedges LM, Brownlie JC, Johnson KN. Wolbachia-mediated antibacterial protection and immune gene regulation in Drosophila. PLoS One. 2011;6(9):e25430.

34. Zhang YK, Ding XL, Rong X, Hong XY. How do hosts react to endosymbionts? A new insight into the molecular mechanisms underlying the Wolbachia-host association. Insect Mol Biol. 2015;24(1):112.

35. Zug R, Hammerstein P. Wolbachia and the insect immune system: what reactive oxygen species can tell us about the mechanisms of Wolbachia-host interactions. Frontiers in microbiology. 2015;6:1201.

36. Cho KS, Lee J, Yoo J, Chung J. Diptericin-like protein: An immune response gene regulated by the anti-bacterial gene induction pathway in Drosophila. Gene. 2001;271(2):233-8.

37. Patrnogic J, Leclerc V. The serine protease homolog spheroide is involved in sensing of pathogenic Gram-positive bacteria. Plos One. 2017;12(12):e0188339.

38. Royet J, Gupta D, Dziarski R. Peptidoglycan recognition proteins: modulators of the microbiome and inflammation. Nat Rev Immunol. 2011;11(12):837-51.

39. Wilmes M, Meier K, Schiefer A, Josten M, Otten CF, Klöckner A, Henrichfreise B, Vollmer W, Hoerauf A, Pfarr K. AmiD Is a Novel Peptidoglycan Amidase in Wolbachia Endosymbionts of Drosophila melanogaster. Front Cell Infect Microbiol. 2017;7:353.

40. Breton C, Snajdrová L, Jeanneau C, Koca J, Imberty A. Structures and mechanisms of glycosyltransferases. Glycobiology. 2006;16(2):29r-37r.

41. Sebastian Grönke 1 GM. Hirsch J, Sonja Fellert. Dual Lipolytic Control of Body Fat Storage and Mobilization in Drosophila. Plos Biology. 2007; 5(6):e137.

42. Subramanian M, Jayakumar S, Richhariya S, Hasan G. Loss of IP3 receptor function in neuropeptide secreting neurons leads to obesity in adult Drosophila. BMC Neurosci. 2013;14:157.

43. Ashburner M, Golic K, Hawley R. Drosophila. A Laboratory Handbook. Trends Genet. 1989;41(5638):535-6. 
44. Paraskevopoulos C, Bordenstein SR, Wernegreen JJ, Werren JH, Bourtzis K. Toward a Wolbachia multilocus sequence typing system: discrimination of Wolbachia strains present in Drosophila species. Curr Microbiol. 2006;53(5):388-95.

45. Koukou K, Pavlikaki H, Kilias G, Werren JH, Bourtzis K. Influence of antibiotic treatment and Wolbachia curing on sexual isolation among Drosophila melanogaster cage populations. Evolution. 2006;60(1):87-96.

\section{Figures}

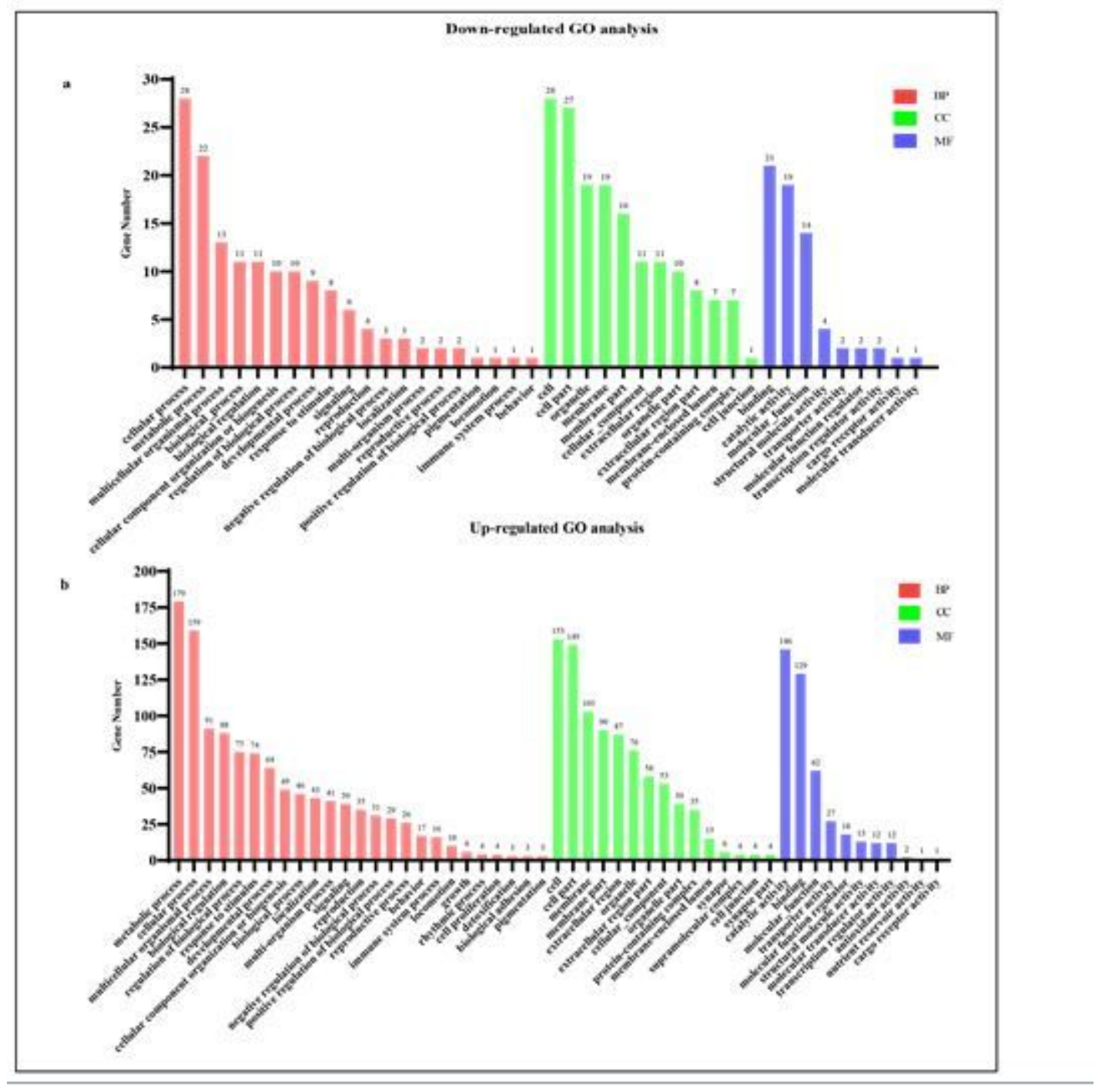

Figure 1

Functional GO enrichment analysis of the down-regulated genes(a) or up-regulated genes(b) in Wolbachia-infected Drosophila melanogaster adult testis compared to Wolbachia-free sample. 


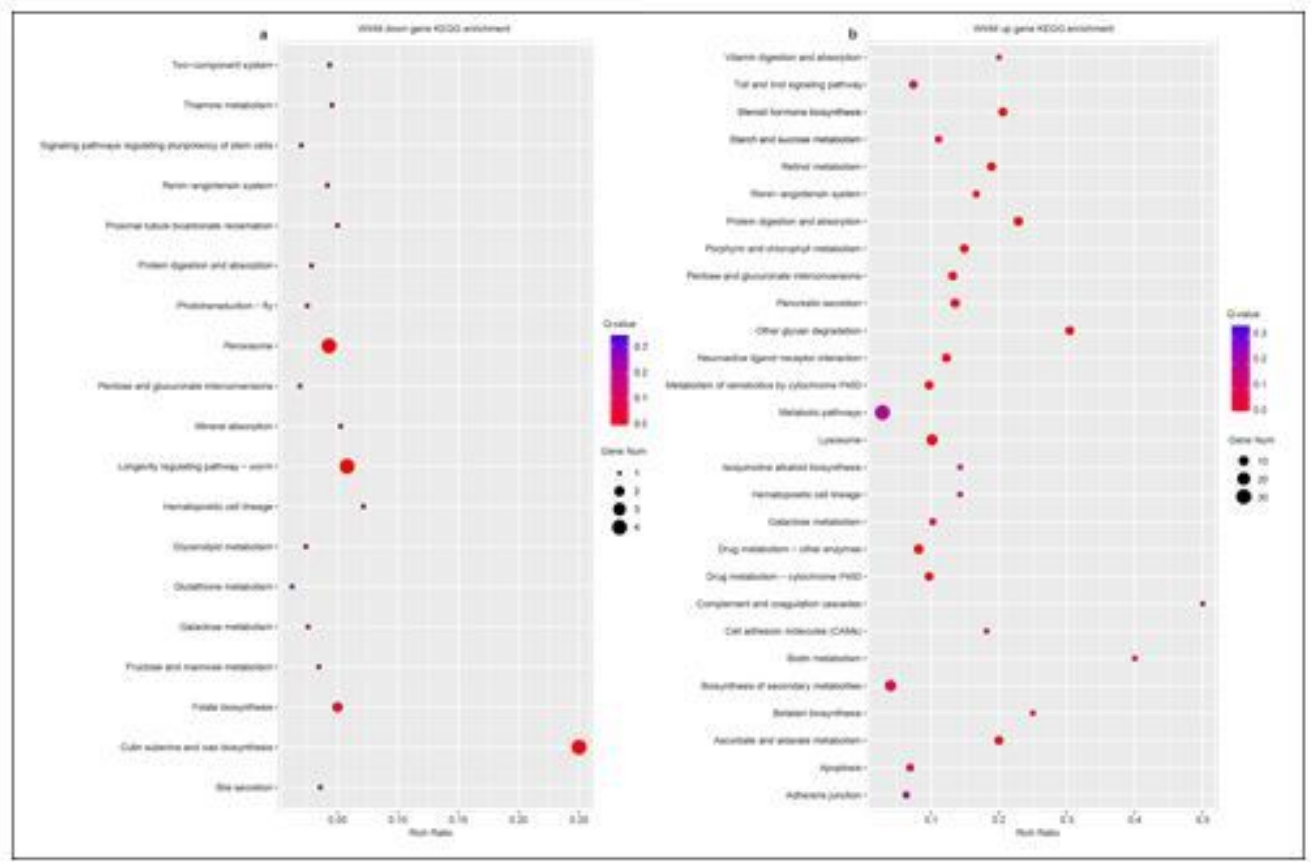

Figure 2

KEGG enrichment analysis on the significantly differentially expressed genes between the adult testis of Wolbachia-infected (WInM) and Wolbachia-free (WUnM) Drosophila melanogaster. The results for genes down-regulated in WInM is shown in (a) and up-regulated genes in WInM is shown in (b).

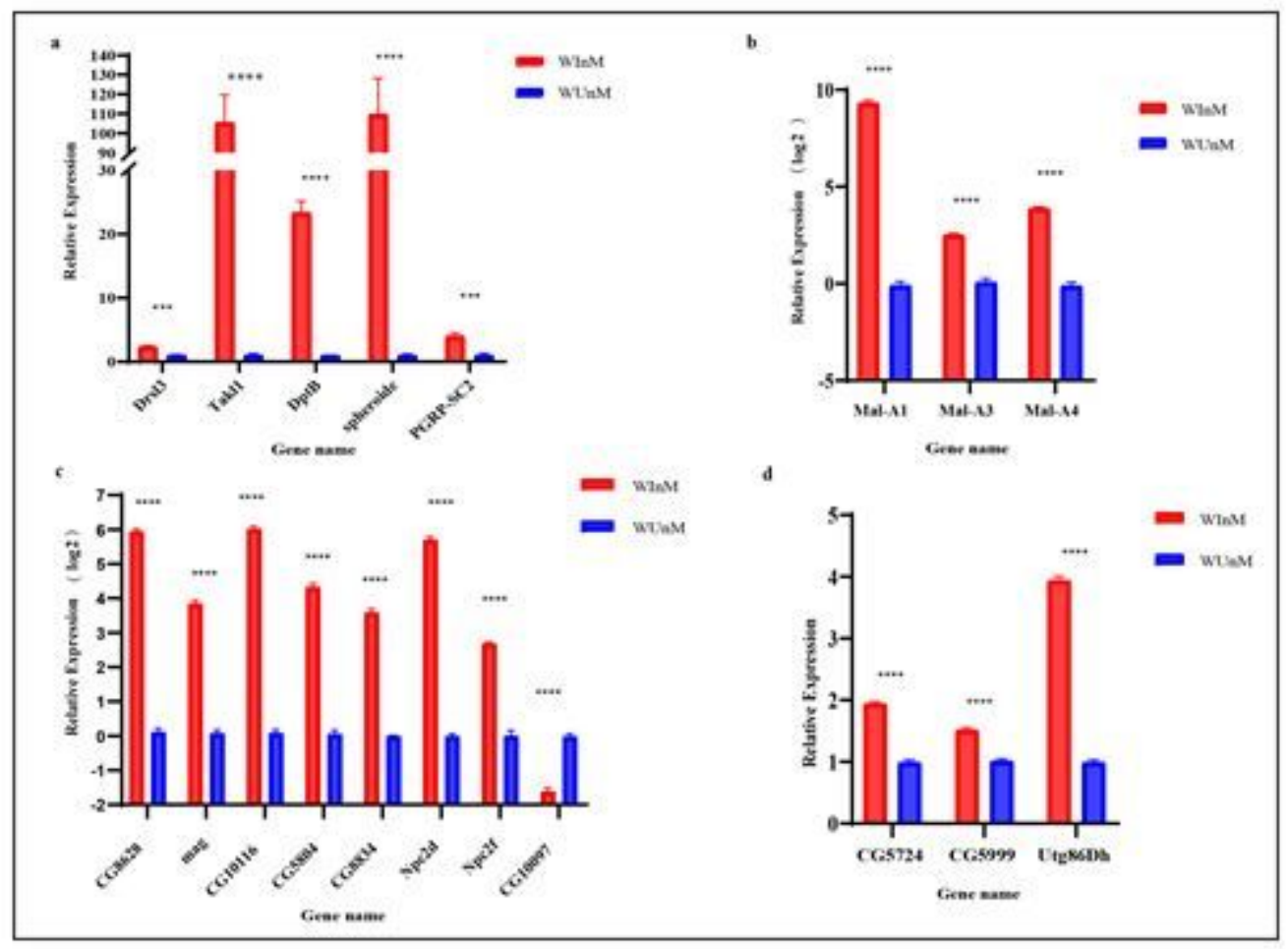

Figure 3

Quantitative PCR validation on some of the significantly different expressed genes between Wolbachiainfected and Wolbachia-uninfected Drosophila testis in innate immune response(a); carbohydrate 
metabolism(b,d) and lipid metabolism(c). Statistical signifificance was determined with multiple t-test in

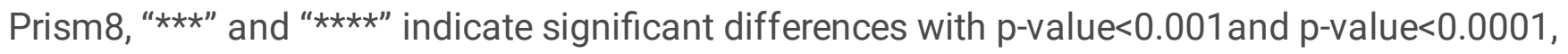
respectively, $n=3$.

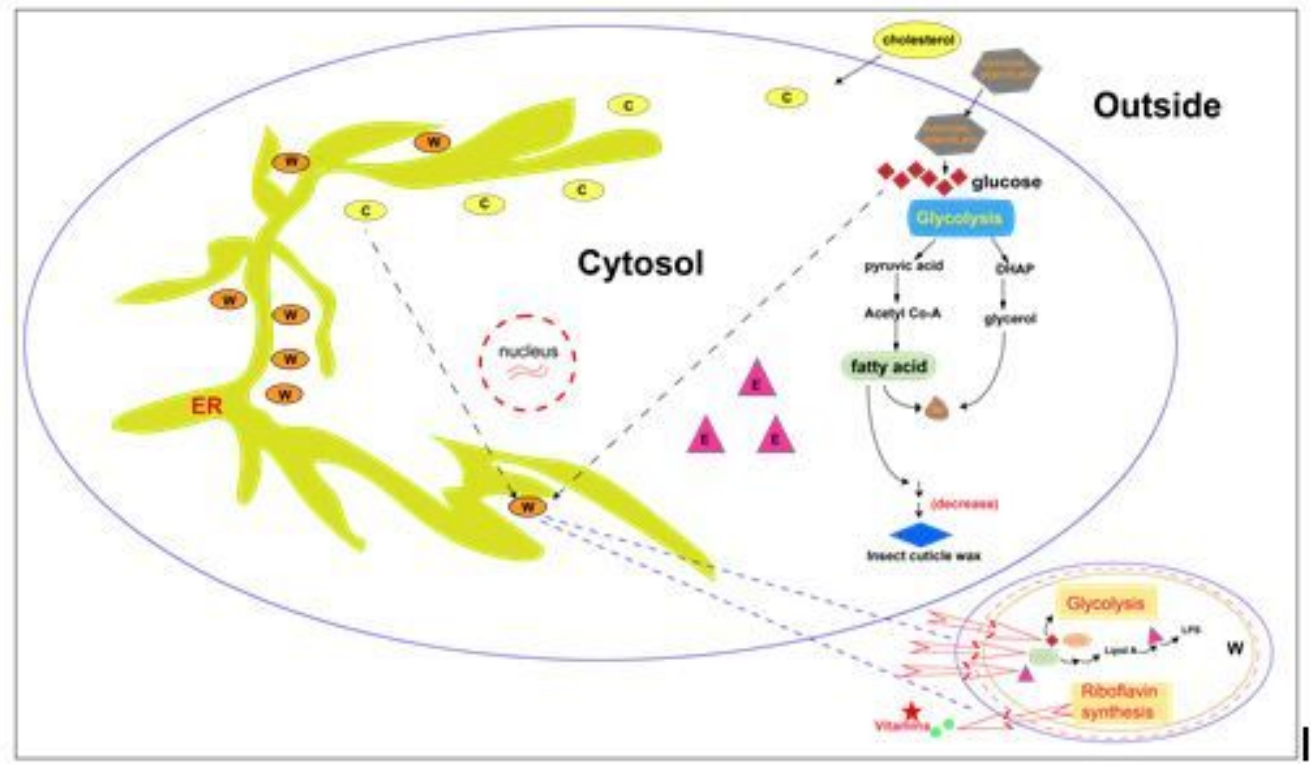

\section{Figure 4}

Schematic diagram of the possible metabolic interactions between Wolbachia and host. By the picture, we want to show how host cells absorb cholesterol (Labeled as C in yellow ovals), starch, surose and other nutrients from the outside; Starch and sucrose are hydrolyzed to glucose (red diamond), which then participates in glycolysis to generate fatty acids and other substances. On the other side, Wolbachia (Labeled as W in orange ovals), mainly located in Endoplasmic reticulum (ER), plunder the glucose of the host, resulting in the acceleration of hosts' glucose metabolism, but inhibition of the synthesis of insect cuticle wax (blue diamond) with fatty acids; Meanwhile, Wolbachia also obtain cholesterol, fatty acids and other substances from the host. The plundered glucose is used for the glycolysis of Wolbachia, and fatty acids are used to synthesize LPS under the action of the host glycosyltransferase (Labeled as E in pink triangle). In return, Wolbachia provides vitamins for the host (Marked with a red star) [23]. The solid lines with arrow indicate the biological reaction process, the dotted lines with arrow indicate that Wolbachia compete with host for some metabolic resources, and the hollow arrows indicate the processes of material exchange among extracellular environment, the host cell, and Wolbachia. DHAP: Dihydroxyacetone phosphate.

\section{Supplementary Files}

This is a list of supplementary files associated with this preprint. Click to download.

- Additionalfile1.docx 